\section{Analysis of Electrical Phenomena in Membranes and Interfaces by Absorption Changes}

IN several biological systems electrical phenomena across membranes of $100 \AA$ thickness are important. As yet thero has been no straightforward way of measuring electrical phenomena in such membranes. If these membranes are activated, a potential difference of about $100 \mathrm{mV}$ is usually built up across them, corresponding to a field strength of about $10^{5} \mathrm{~V} / \mathrm{cm}$.

Fields of this strength can affect several properties of the molecules in the membrane. For instance, they can change electronic absorption within the molecules (electrochroism ${ }^{1}$ ). This would cause absorption changes of the order of 0.1 per cent, which would follow the field changes without any delay.

Absorption changes of this order cannot be detected directly, especially not in short times (for example, $10 \mathrm{~ns}$ ), because the noise would totally mask the signal. If the absorption changes are, however, repeated $n$-fold and averaged, the signal-noise ratio increases proportionately to $\sqrt{ } n$. In this way absorption changes of 0.1 per cent have been measured with a time resolution down to the $10 \mathrm{~ns}$ range by averaging about 1,000 signals. Details of the measuring techniques are summarized in ref. 2.

If biological membranes of $100 \AA$ thickness are pigmented intrinsically or stained artificially and then repeatedly activated, this method can be used to measure electrical field changes across the membranes. In the same way electrical currents or displacements which change the field can be detected. Thus electrical field changes and ion fluxes in photosynthesis have been analysed. in the membrane of thylakoids ${ }^{3}$. For a summary see ref. 4.

Our method may also be a sensitive tool for the analysis of electric phenomena in other biological membranes, for example of nerves, muscles, mitochondria and photoreceptors. The method may also be useful for the analysis of artificial membranes and interfaces.

W. JUNGE

Max-Volmer-Institut,

H. T. WITT

I. Institut für Physikalische Chemie,

Technische Universität,

Berlin.

Received February 27; revised April 18, 1969.

${ }^{1}$ Labhard, H., in Adv. in Chem. Phys., 13 (Interscience, London, 1967).

2 Witt, H. T. in Fast Reactions and Primary Processes in Chemical Kinetico (edit by Claesson, S.) 81 (Interscience, New York, Reactions) (edit. by Colowick, S. P., and Kaplan, N. O.) (Academic Press, New York, in tho press).

${ }^{3} J$ unge, W., and Witt, H. T., Z. Naturforsch., 23b, 244 (1968).

4 Witt. H. T. Rumberg, B. and Junge, W., Nineteenth Mosbach Kolloquium, 262 (Springer-Verlag, Berlin, 1968).

\section{BIOLOGICAL SCIENCES}

\section{Identification of Human Trophoblast in vitro}

WHILE investigating the antigenicity of human trophoblast and its possible role in the toxaemias of pregnancy, we wished to set up pure tissure cultures of trophoblast. Placental tissue in explant and monolayer culture gives rise to a mixed population of cells, the identification of which is difficult because they undergo morphological and physiological changes in vitro. Existing methods for identifying trophoblast in vitro include cytology ${ }^{1,2}$, enzyme histochemistry ${ }^{1}$ and the demonstration of chorionic gonadotrophin production ${ }^{3,4}$. Thiede $^{1}$ distinguished three cell types in cultures of chorionic villi: fibroblasts, epithelioid cells and giant multinucleated cells. $\mathrm{He}_{e}$ suggested that these originated from mesenchyme, cytotrophoblast and syneytio-trophoblast respectively, but emphasized the unreliability of morphological criteria. The histochemical localization of alkaline phosphatase in epithelioid and multinucleate cell types ${ }^{1}$ is considered compatible with an origin from trophoblast in view of the distribution of this enzyme in the placenta found by several workers ${ }^{5-9}$. Soma, Ehrmann and Hertig ${ }^{3}$ found that the gonadotrophic activity of the culture medium declined in concert with the disappearance of giant multinucleated cells, suggesting that these might be trophoblastic in origin.

We have attempted a more specific identification of trophoblast using the sandwich fluorescent technique with specific anti-trophoblast serum raised in mice, and a fluorescent-labelled anti-mouse serum ${ }^{10}$.

Placentae of gestational age 12-14 wecks were obtained at the time of therapeutic termination of pregnancy. 'The tissue was cultured using Thiede's method ${ }^{1}$. Villi were dissected off the chorionic plate, washed in Hanks balanced salt solution and incubated at $37^{\circ} \mathrm{C}$ for $30 \mathrm{~min}$ with either 0.1 per cent or 0.2 per cent trypsin. The suspension was filtered through gauze and the cells were collected by centrifugation at $800 \mathrm{r} . \mathrm{p} . \mathrm{m}$. for $8 \mathrm{~min}$. The cells were resuspended in medium 199 containing 10 per cent pooled human serum and $0.5 \mathrm{ml}$. of suspension added to stationary culture tubes containing coverslips.

Coverslips wero removed after 7 days, dried in air at room temperature for $3 \mathrm{~h}$ and the cultures were stained using the "sandwich" fluorescent technique. Cultures were washed with buffered saline and incubated for 30 min at room temperature with mouse anti-serum in dilutions of $1: 8,1: 16,1: 32$. The anti-serum was washed off the section with three changes of buffered saline. Goat anti-mouse globulin labelled with fluorescein isothiocyanate was used to stain the cultures for $30 \mathrm{~min}$ at room temperature. After washing with three changes of buffered saline the coverslips were mounted in buffered glycerol and examined with a Leitz fluorescence microscope. The cultures were subsequently re-stained with haematoxylin and eosin and examined with the light microscope.

Specific fluorescence was shown by the large syneytial masses present in the cultures, fluorescence being confined to the cytoplasm (Fig. 1). Stained with haematoxylin and eosin such areas of fluorescence appeared as irregularly shaped cells containing many nuclei (Fig. 2). Cultures prepared by disaggregation with $0 \cdot 1$ per cent trypsin contained only this type of cell. Cultures prepared by disaggregation with 0.2 per cent trypsin contained, in addition to syncytial cells, epithelioid cells showing specific fluorescence, and typical fibroblasts and red blood cells which showed autofluorescence only.

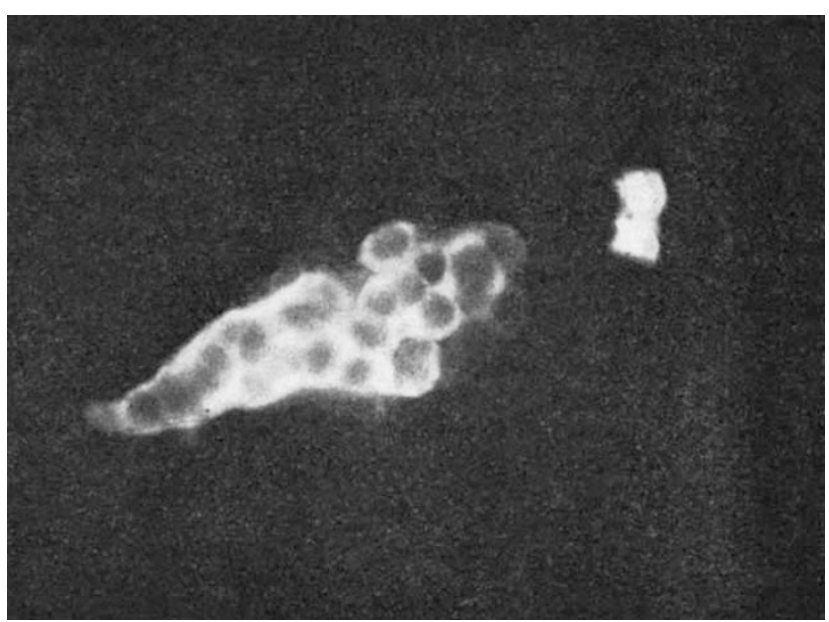

Fig. 1. Ultraviolet photomicrograph of syncytium. ("Sandwich" fluorescent preparation with anti-trophoblast serum, $\times 300$.) 